\title{
Radiologie up (to) date - auch 2017 wieder ein großer Erfolg
}

Das Radiologie Update fand 2017 am 31. März und 01. April wieder in Ulm statt. Wie bereits im Vorjahr konnte erneut die Anzahl der Teilnehmerinnen und Teilnehmer gesteigert werden, gleichermaßen aus Praxis und Klinik. Dies ermöglichte einen Umzug in den großen Saal des Kongresszentrums, zudem mit einer großzügigen Ausstellerfläche. Sowohl Vortragsfolien wie zusammenfassende und wertende Skripte standen den Teilnehmerlnnen zum „Download“ bereit. Die Bereiche Neuroradiologie und Muskuloskelettale Radiologie wurden diesmal ihrer besonderen Bedeutung entsprechend durch jeweils zwei Vorträge hervorgehoben. Für das „Hot Topic“ Kontrastmittel konnte Herr PD Dr. Alexander Radbruch aus Heidelberg als international anerkannter Experte für einen Übersichtsvortrag gewonnen werden. In bewährter Weise beleuchtete RA Markus Henkel vom Berufsverband Deutscher Radiologen aktuelle Aspekte der Berufspolitik. Professor Michael Forsting aus Essen brachte in brillanter Weise die Radiologie auf den Punkt. Erstmalig wurde das Radiologie Update gemeinsam mit medupdate veranstaltet. Frühlingshafte Temperaturen erlaubten den Beginn des Gesellschaftsabends ins Freie zu verlegen, der in gewohnt lockerer kollegialer Atmosphäre verlief.

Inhalte der neuen WHO-Klassifikation von Hirntumoren sowie deren Auswirkungen auf die radiologische Diagnostik stellte Bernd Schmitz aus Ulm im ersten Vortrag zum Themenbereich Neuroradiologie vor. Zweiter Aspekt seines Vortrags waren zerebrale Inflammationserkrankungen. Neuerungen in der Stadieneinteilung beeinflussen deren Erfassung und Differentialdiagnose. Die Studienlage bezüglich der Abgrenzung der Amyloidangiopathie zur
Mikroangiopathie wurde zudem vorgestellt.

Die vaskuläre Neuroradiologie mit Diagnostik und Therapie der zerebralen Ischämie stand im Fokus des zweiten Vortrags Neuroradiologie von Mirko Pham aus Würzburg. Basierend auf der Darstellung der pathophysiologischen Grundlagen wurde die Studienlage sowohl bezüglich der Diagnostik wie der Therapieoptionen zusammengefasst, an Patientenbeispielen diskutiert und mit den aktuellen Empfehlungen abgeglichen. Die Besonderheiten der stroke mimics und Strategien zu deren Abgrenzung wurden diskutiert.

Christina Schraml aus Rosenheim fasste die beiden Themenkomplexe Leber und Gastrointestinaltrakt zusammen. Neben der Wertigkeit leberspezifischer MRT-Kontrastmittel für Differentialdiagnosen wurden zentrale Protokollfragen dargestellt. Die Bedeutung der sogenannten LI-RADS-Kategorisierung stellte einen zweiten Schwerpunkt dar. CT-Techniken bei der Fragestellung „Dünndarmischämie“, Protokoll-Optimierungen bei der MR-Enterografie und wesentliche Punkte der ESGAR Guidelines wurden zudem dargestellt.

Die aktuelle Studienlage zu den Themenkomplexe Tumoren des Pankreas und des Beckens stellte Markus Juchems aus Konstanz dar. Neben Protokollempfehlungen zur Detektion und Verlaufsbeobachtung zystischer und neuroendokriner Pankreastumoren wurden neue technische Ansätze wie die Perfusions-CT erörtert. Ein weiterer Aspekt waren Studien zur Autoimmunpankreatitis. Bei Beckentumoren, wie des Uterus und des Ovars, verbessert die MRT-Diffusionsbildgebung die Möglichkeiten zu
Detektion, Differentialdiagnose und Verlaufsbeurteilung.

Neuerungen bezüglich der Herz- und Gefäßdiagnostik wurden von Jörn Sandstede aus Hamburg zusammengefasst. Implikationen der detaillierten Plaquediagnostik mittels des Herz CTs sowie die neuen Versorgungsleitlinien zur chronischen KHK wurden dargestellt und anhand von Patientenbeispielen diskutiert. Auswirkungen der CAD-RADS-Klassifikation auf Befunderstellung- und Interpretation, technische Weiterentwicklungen der zeitaufgelöste MR-Angiografie zur Gefäßdiagnostik und Einsatzmöglichkeiten des T1- und T2-Mappings wurden vorgestellt.

Daniel Spira aus Heidelberg fasste neue Aspekte bezüglich der Diagnostik bei Lungenfibrose und chronisch obstruktiver Lungenerkrankung zusammen. Durch die Einführung von Lung-RADS sowie der neuen Empfehlungen der Fleischner-Society haben sich Änderungen für das Management von Lungenrundherden ergeben. Protokolloptimierung der CT-Diagnostik bei Lungenembolieverdacht und die Bedeutung des „tree in bud“ Phänomens wurden zudem diskutiert.

Für die Kinderradiologie fasst Meinrad Beer aus Ulm die Studienlage zusammen. Neben der zunehmenden Bedeutung des kontrastmittelverstärkten Ultraschalls wurden Neuerung bei Dosisreferenzwerten, Dosisreduzierung von $\mathrm{CT}^{\prime}$ 's und neue Ansätze des Lungen MRT's dargestellt. Studien zur Pankreatitis, Appendizitis- und Kolitis-Diagnostik wie zur fetalen MRT wurden vorgestellt. Die DVT-Technik könnte bei Kindern mit Extremitätentraumata zunehmend eingesetzt werden. 
Katja Siegmann-Luz aus Berlin stellte die Neuerungen für die Mammadiagnostik zusammen. Neben der aktuellen deutschen Version der BI-RADS-Klassifikation wurden Ergebnisse und Erfolge des MammografieScreenings dargestellt. Im Folgenden wurden die Bedeutung der Brustdichte als Risikofaktor und Erfordernisse einer ergänzenden Bildgebung sowie das Potential der MR-Mammografie bezüglich reduzierter Reoperationsraten und verbessertem Outcome diskutiert.

\section{Bandstrukturen}

Wolfgang Fischer aus Augsburg stellte neueste Ergebnisse zur muskulosklettalen Bildgebung der unteren Extremität dar. Hier spielt insbesondere die Differenzierung zwischen traumatischen Veränderungen und Degeneration eine ganz wesentliche Rolle. Weitergeführt zum Vorjahr wurde die Diskussion zu Bandstrukturen im anterolateralen Abschnitt des Kniegelenkes. Funktionelle Beeinträchtigungen im Hüftgelenk und entzündliche Veränderungen der Wirbelsäule sowie der Ileosacralgelenke bildeten weitere Aspekte seines Vortrages.

Die obere Extremität wurde von Reto Sutter aus Zürich vorgestellt. Für den Schulterbereich wurden die MR-Neurografie des Plexus brachialis, knöcherne 3D-Rekonstruktion basierend auf MRT-Bildern und neue Ansätze zur Beurteilung und Quantifizierung der Rotatorenmanschette vorgestellt. Auf CT/MRT-Bildern basierende computerassistierter OP-Planung für das Kompartment-Syndrom des Ellbogengelenkes, mittels Traktions-MRT durchgeführte Dar- stellungen von Sehnenverletzungen am Handgelenk wurden abschließend dargestellt.

Im „Hot-Topic“ Vortrag fasste Alexander Radbruch aus Heidelberg die aktuelle Studienlage hinsichtlich Gadolinium-Ablagerungen im menschlichen Gehirn zusammen. Aktuelle pathophysiologische Modelle der möglichen Mechanismen zur Ablagerung im Gehirn wurden in anschaulicher Weise skizziert. Eine ausgewogene Darstellung der verschiedenen Argumente rundeten den, wie zu erwarten, stark diskutierten Beitrag ab.

Michael Uder aus Erlangen stellte die aktuelle Studienlage zur Uroradiologie vor. Die Bedeutung des multiparametrischen MRT's, insbesondere in Verbindung mit einer MR-geführten transrektalen Ultraschallbiopsie, beim Prostatakarzinom und die Anwendbarkeit der zweiten Version der PIRADS-Klassifikation waren wesentliche Themen. Verbesserungen der diagnostischen Trennschärfe bei der ätiologischen Zuordnung von Nebennierenraumforderungen sowie beim Management solider Nierenläsionen wurden vorgestellt. Komplizierte Nierenzysten stellen weiterhin eine Herausforderung für CT, MRT und CEUS dar, können aber bei geschickter Kombination verschiedener Verfahren sicher eingeordnet werden.

Intensive und spannende Diskussionen ergaben sich wiederum beim Vortrag von Markus Henkel, Berufsverband der Deutschen Radiologen, München, zur Thematik der Berufspolitik. Der Stand zur Novellie- rung der Strahlenschutzvorschriften, insbesondere zu den Aspekten Früherkennung sowie Teleradiologie, Änderungen in der Musterweiterbildungsordnung bildeten wichtige Aspekte. Bezüglich Bedarfsplanung, EBM und Gebührenordnung der Ärzte liegen aktuelle Vorschläge vor, die teils gesetzlich noch umgesetzt werden müssen. Hier sind entscheidende Weichenstellungen zu erwarten, die vorgestellt wurden.

Zum zweiten Mal gestaltete Michael Forsting aus Essen den Highlight-Vortag. Neben der Vorstellung komplexer wie spannender Fallberichte führte er die Diskussion zur Thematik „Künstliche Intelligenz" und der Anwendung in der Radiologie fort. Automatische Detektionssysteme werden entscheidend den Arbeitsalltag in der Radiologie verändern. Möglicherweise eine Chance, um den immer größer werdenden quantitativen wie qualitativen Ansprüchen der Zuweiserinnen und Zuweiser gerecht zu werden.

Auch diesmal war das Radiologie Update durch den besonderen Mix aus spannenden „state-of-the-art“ Vorträgen, anregenden Diskussionen und dem kollegialen Austausch geprägt. Auf ein Wiedersehen beim Radiologie Update 2018 freuen wir uns.



Prof. Meinrad Beer Ulm 